\title{
Comme il nous plaira
}

\section{Michèle Le Doeuff}

\section{(2) OpenEdition}

Journals

Édition électronique

URL : http://journals.openedition.org/shakespeare/2958

DOI : 10.4000/shakespeare.2958

ISSN : 2271-6424

Éditeur

Société Française Shakespeare

Référence électronique

Michèle Le Doeuff, «Comme il nous plaira », Actes des congrès de la Société française Shakespeare [En ligne], 33 | 2015, mis en ligne le 10 mars 2015, consulté le 06 juin 2020. URL : http://

journals.openedition.org/shakespeare/2958; DOI : https://doi.org/10.4000/shakespeare.2958

Ce document a été généré automatiquement le 6 juin 2020

(c) SFS 


\title{
Comme il nous plaira
}

\author{
Michèle Le Doeuff
}

1 Dominique Goy-Blanquet a bien du mérite à donner la parole à quelqu'un qui n'a jamais suivi un quart d'heure de cours sur Shakespeare de sa vie; et vous avez du mérite à être là. Mais telle serait précisément la raison de ma présence : je vais tâcher de montrer que la popularité de Shakespeare aujourd'hui tient en partie à une réception non codée, hors des circuits officiels, qui a poussé comme les herbes des champs, les fleurs qui croissent le long des voies ferrées, grâce à l'engouement au premier degré d'un public peu convenu, sauvageon, qui n'aime que ce qu'il aime et pourrait prendre à la lettre l'invitation de voir le théâtre " as you like it », donc comme il nous plaira, " as you wish », comme on veut, en toute liberté.

2 L'aventure commence pour moi quand j'ai une dizaine d'années. Je lis Shakespeare sans trop m'en vanter. Puis surviennent des événements qui me donneront à réfléchir. Le Théâtre municipal de Quimper - dont je suivais assidûment les matinées scolaires proposa Macbeth. Et c'était comme d'habitude, comme pour Corneille, Molière et Racine, une mise en scène un peu littérale, un plateau encombré de décors et de meubles, des effets de caractère, - comme si un même format esthétique pouvait s'appliquer de façon générale à tous les auteurs. Mais la magie ordinaire était là aussi, les velours rouges du vieux théâtre, les trois coups, le rideau qui se lève pour dévoiler une boîte dans laquelle il va se passer quelque chose. Pourtant, et alors que c'était comme d'habitude, ce jour-là, un chahut de potaches se déclencha au parterre, avec rires et cris d'animaux, sporadique d'abord puis si lourd qu'il fallut interrompre la représentation : un comédien s'adressa à la salle, comme contraint de déclarer qu'il y a des gens qui aimeraient pouvoir faire leur métier. Cette parole d'autorité, dite sur un ton de professeur, calma le jeu. C'était bizarre tout de même - car d'ordinaire, la salle entière était fort sage. Comme le parterre était occupé par les élèves des établissements pour garçons, les filles devant se tenir au balcon, on peut à coup sûr y voir une question de genre : les filles ne se sont pas jointes au chahut, sans doute certains garçons non plus. Retenons en tout cas que c'est au sein du groupe masculin que le chahut, indice de rejet primaire, eut lieu. 
3 Pourtant, avec la question du genre, il faut saisir autre chose : quand on leur servait Corneille, Racine ou Molière, tous les garçons se tenaient de façon convenable et policée. Ce jour-là, on leur présentait une pièce ressemblant aux œuvres du programme mais qui n'en était pas, et ils se défoulaient. En ce temps-là, vers 1960, Shakespeare n'apparaissait plus du tout dans l'enseignement secondaire, du moins là où on m'a appris l'anglais. Ont-ils réagi à ce qu'ils ont perçu comme une contrefaçon, ces garçons toujours dociles quand on présentait les auteurs de « la religion de l'État ${ }^{1}$ » mais qui se révélaient chameaux véritables quand on passait en dehors du canonique ? Tandis que les filles... quoi, les filles? Polies en toute circonstance, direz-vous, car élevées ainsi ? Peut-être. Ou, tout au contraire, moins formatées, moins cantonnées dans une norme? Plus assoiffées de découvertes? Vous jugerez peut-être la seconde hypothèse invraisemblable: voyons, au début des années soixante à Quimper? Mais pourquoi pas? Après tout, j'en étais ; je peux au moins répondre de ma propre réaction, qui fut loin d'être un rejet pur et simple : j'étais contente d'être venue, bien qu'à la sortie, et comme toujours, la question horrible, redoutable, la pire question qui soit, m'ait traversé l'esprit : est-ce que, tout compte fait, je n'aime pas mieux lire cette pièce? Il arrivait que je penche pour l'affirmative.

Dans le cas de Macbeth, la réponse fut plus nuancée. Sur scène, Lady Macduff et son fils, la douleur mêlée de honte du mari et père, dépassaient de loin mes souvenirs de lecture. L'interprétation avait « sorti » du texte des vibrations dont je ne me doutais pas et qui m'avaient touchée. Si l'on peut extrapoler, à titre d'hypothèse: le public "sauvageon", qui va progressivement aider à construire la très large réputation de Shakespeare en France et ailleurs, serait-il issu - en partie, du moins - d'un public de lecteurs et lectrices, qui a goûté le texte en version imprimée et qui va au théâtre chercher "plus », plus de plaisir, plus d'émotions, plus de compréhension d'une œuvre déjà aimée en tête-à-tête ? Je crois que ce fut le cas d'Aimé Césaire et qu'il a d'abord lu Shakespeare, comme le bon élève autodidacte qu'il était, car les vrais bons élèves savent toujours être autodidactes par surcroît. En tout cas, il a toujours nié avoir découvert Shakespeare à Paris, « ce que Paris m'a apporté, c'est l'Afrique ${ }^{2}$ »; on est en droit d'en inférer que ce fut à Fort-de-France, dans une bibliothèque familiale, scolaire ou municipale, qu'il a rencontré l'auteur avec lequel plus tard il croisera le fer puis se réconciliera. Les livres voyagent plus aisément que les troupes et sont toujours plus accessibles.

5 Une Tempête, la relecture critique de La Tempête qu'Aimé Césaire va offrir au public, n'a rien à voir avec le chahut - conformiste - de nos camarades garçons qui, ayant déjà intériorisé une définition officielle de ce qui compte comme théâtre, donnaient de la voix contre tout ce qui s'écartait de ladite définition. En revanche, ce chahut mérite d'être rapproché de celui qui eut lieu en 1822 , à l'occasion d'une représentation d' Othello à Paris ainsi que d'une autre histoire, historiquement plus proche et tout aussi pénible: en 1945, Charles Dullin monte Le Roi Lear, la critique l'éreinte, et Simone de Beauvoir monte au créneau pour défendre Dullin en contre-attaquant. Or par rapport aux études shakespeariennes, Simone de Beauvoir est bien une sauvageonne. Témoignage de Dullin lui-même sur la mauvaise presse à laquelle il fut confronté : «le spectacle présenté chez moi était jugé, condamné et quasiment interdit. Le choc en retour fut l'abstention du gros public et la méfiance chez les autres. Ce n'est que la réclame parlée [comprenons : la publicité à la radio] et quelques articles de fond me rendant justice qui permirent au public de se regrouper ${ }^{3}{ }^{~}$. 
6 L'article de Simone de Beauvoir, intitulé «C'est Shakespeare qu'ils n'aiment pas » et publié dans Action, est au nombre des articles de fond rendant justice à Dullin. De fait, elle cherche à analyser l'adversaire en ouvrant une polémique qui constitue un décryptage politique de l'événement. L'article s'ouvre sur un rappel du «temps où Alain Laubreaux et ses congénères " sévissaient, temps pas si éloigné que cela, et Beauvoir note «Il semble qu'ils aient, hélas! créé une tradition ${ }^{4}$ ». L'accusation est grave - Laubreaux vient de s'exiler en Espagne et sera condamné à mort par contumace en 1947 pour faits de collaboration. Elle voit l'éreintage de la mise en scène de Dullin comme politique, il est d'ailleurs à noter qu'elle publie sa contre-attaque dans Action, un journal sorti de la clandestinité moins d'un an auparavant et se réclamant de « la France combattante ${ }^{5} »$. De même qu'en 1822, des jeunes libéraux avaient accusé Shakespeare d'avoir traversé la Manche dans les bottes de Wellington - comme si le malheureux Will avait quelque chose à voir avec Waterloo - de même, devine-t-on à lire Beauvoir, il est vu comme arrivé dans les chalands du Débarquement, par certains qui, en 1945, craignaient peu ou prou les purges. Une différence cependant: en 1822, les détracteurs s'en prennent directement à Shakespeare ; en 1945, le rejet est plus retors les critiques s'en prenant surtout à la mise en scène de Dullin. C'était plus facile ! Car les gens qui lisent les critiques de théâtre n'ont pas encore vu le spectacle, on peut donc leur dire n'importe quoi à ce sujet, alors que ceux qui ont déjà lu et aimé la pièce ne seraient pas prêts à s'en laisser conter.

7 Deux arguments sont à retenir: selon Beauvoir, les critiques ne parlent pas du spectacle lui-même, "c'est peu de dire qu'ils se préfèrent à l'objet devant lequel ils devraient pourtant s'effacer: l'objet n'existe pas pour eux.» Sans doute faut-il comprendre : ils aiment écrire des philippiques et ils ont trouvé dans la mise en scène de Lear une occasion commode d'assouvir ce penchant. Mais sous cette indifférence apparente, issue de l'égocentrisme, Beauvoir déchiffre une hostilité radicale: «Ils feignent d'attaquer Dullin, et dans le fond c'est à Shakespeare qu'ils en veulent. » L'un s'attache aux casques, aux robes des guerriers, à la forme d'un bouclier, et pas pour en dire du bien. Un autre lâche que «[c]e n'est pas encore cette présentation qui fera accepter Le Roi Lear à ceux qui osent avouer qu'ils le trouvent ennuyeux. » Tiens! Et Beauvoir de conclure qu'il s'agit du «détestable héritage d'un passé qui doit être balayé », en clair, du nationalisme à la Pétain, un nationalisme qui comporte aussi une grosse dose de ressentiment vis-à-vis d'un auteur étranger, d'un auteur du dehors, qui a le front de prétendre exister. "C'est à Shakespeare qu'ils en veulent, c'est Shakespeare qu'ils n'aiment pas». Le démontage, par Simone de Beauvoir, de l'éreintage du Roi Lear par la critique théâtrale en 1945 correspond à ce que j'ai senti à Quimper au début des années soixante : le rejet de tout ce qui peut apparaître comme " off " - hors norme - rejet renvoyant à l'étroitesse d'esprit et à quelque chose de plus grave. Car enfin, à quoi cela sert-il d'avoir étudié Corneille et Molière, si cela ne vous a pas donné le goût de l'œuvre théâtrale en général, donc l'aptitude à goûter aussi d'autres œuvres, Shakespeare ou Kleist ou Sophocle? Au reste, Shakespeare n'était pas à Quimper la seule victime de la clôture esthétique. Dans la classe d'à-côté, on ne faisait pas de latin et donc on étudiait davantage de littérature française. La Princesse de Clèves fut, paraît-il, qualifiée par l'enseignante elle-même de "cucul-la-praline», ce qui n'était pas gentil! Il y eut aussi un Corneille présenté à la télévision (dont je ne peux rien dire car on n'avait pas la télévision à la maison). Le corps enseignant fit campagne contre cette programmation, une campagne à la limite de l'appel au boycott. «Maintenant, il y a des gens qui vont dire qu'ils connaissent Corneille, alors que ce 
n'est pas vrai : ils ne connaissent pas Corneille ». Mais à Paris comme à Quimper :j'ai un jour fait hurler des amis de Lettres modernes en évoquant une lecture freudienne de Hamlet. Là, apparemment, un Shakespeare sacralisé, devant être maintenu à l'abri de la psychologie des profondeurs, s'était installé. On se permettra de dire que ce n'est guère mieux.

8 Le travail de balayage du passé détestable, Beauvoir l'imagine comme passant par une réforme de la critique théâtrale. Les critiques «ne semblent pas soupçonner quelles responsabilités reposent sur leurs épaules : l'artiste a besoin d'un public, et le public a besoin de guides sûrs ${ }^{6}$ ». Sans doute est-ce vrai, quand on pense en termes de "gros public ", pour reprendre l'expression de Dullin, et à la "méfiance ", en effet assez courante, de ceux qui pensent être hors du commun mais qui craignent d'aimer ce qui n'a pas été approuvé par les connaisseurs. Cependant, et malgré tout le respect amical que j'éprouve pour notre Simone, on ne peut lui donner raison qu'à moitié. Mon premier argument sera Claude Barma, mon second un Troilus et Cressida inoubliable à l'École Normale, mon troisième la compagnie "The Northern Broadsides", et vous complèterez la liste vous-mêmes si vous voulez, en y ajoutant par exemple Augustin d'Humières à Meaux ou les enfants d'un camp de réfugiés en Jordanie ${ }^{7}$. Au reste, on peut compter sur Simone de Beauvoir pour se juger plus sévèrement que nécessaire. Dans La Force des choses, elle revient sur la question, en proposant un grand éloge de Simone Jollivet, Dullin et Ariane Borg, mais de son propre texte, elle dit: «Je n'en espérais pas grand-chose et il n'en résulta rien $^{8} »$. Allons : elle aura au moins montré qu'on peut critiquer la critique, par exemple théâtrale, et que celle-ci n'a pas toujours le dernier mot. Ce n'est pas rien. Mais peut-être faut-il y percevoir l'indication d'un fait qui mérite d'être relevé: Simone n'a jamais réussi à dire quoi que ce soit de Shakespeare lui-même. Une brève référence dans Le Deuxième Sexe, une autre, plus éclairante, dans un chapitre (non retenu) d'un brouillon de L'Invitée 9 . On peut parler de mutisme. D'où une question: à quelles conditions une femme peut-elle, des femmes peuvent-elles, tenir une parole sur l'œuvre ou l'univers de Shakespeare? Qui faut-il être comme femme pour entrer dans l'interprétation des relations indébrouillables d'un père avec ses trois filles ou d'ailleurs des ambiguïtés sexuelles des femmes travesties en hommes? Cette question, je l'adresse aux shakespeariennes ici présentes.

En tout cas, l'idée d'un public qui aurait besoin d'être "guidé », idée qui fonde la nécessité d'une critique théâtrale plus éclairée, plus honnête, peut sans doute se plaider au nom du réalisme: monter une pièce représente un gros investissement financier. Néanmoins, cette idée est loin de rendre compte de l'apport d'amoureux non officiellement agréés, sauvages et audacieux, qui ont su communiquer une passion. Vacances de Noël 1962: des voisins m'invitent à venir voir chez eux La Nuit des rois, montée pour la télévision par Claude Barma. Ce fut une révélation, car c'était mille fois mieux que le petit théâtre intérieur que la lecture avait pu me procurer. Pas de boîte ici, un espace blanc dont on ne voyait pas les contours, un espace sans fin, peu de décors, simplement des praticables; du coup, le corps des comédiens et leur costume ressortent et remplacent le décor comme visuel, leur corps et leur visage, sur lequel le gros plan permet de guetter les émotions les plus fugitives. Et dans ce dépouillement extrême, le texte, tout le texte, se fait entendre, ce qui restera une des conditions de mon plaisir. Subjuguée par Robert Hirsch en Feste, éblouie par Geneviève Page en Viola, j'ai vécu une conversion esthétique. Bien plus tard, je découvrirai une interview de Pierre-Aimé Touchard à propos du Macbeth de Claude Barma, et disant au public: "Vous êtes les seuls juges", "Un seul but: vous plaire», - explicitation à peine 
nécessaire. Des spectateurs, des lecteurs aussi d'ailleurs, le sentent bien quand ils sont mis en situation de juges et qu'on cherche avant tout à leur plaire ou à leur parler, donc quand la production cherche son centre de gravité en dehors d'elle-même, c'est-à-dire en nous. Pensons au dernier vers de La Nuit des rois, "we'll strive to please you everyday ", rude condition de vie pour une troupe de théâtre, mais c'est ainsi : il faut plaire et faire plaisir tous les jours.

J'ai avoué avoir vécu ce soir-là une conversion esthétique : on peut monter une œuvre dans la légèreté, la simplicité, avec apparemment peu de moyens, en faisant fond sur le talent et le travail des comédiens. Et rien d'autre. Sans appui préalable : on prend un auteur qui, à l'époque, était peu prisé du public français - on monte une pièce pour un medium encore passablement dédaigné, la télévision. Un double écart, donc, et ça marche. Barma a fait confiance à son public potentiel. Avis aux futurs metteurs en scène : le degré d'estime que vous avez pour votre public, cela se voit toujours. En outre, la pièce est relativement osée, puisqu'on assiste en principe à la virilisation progressive d'une jeune fille: Viola demande qu'on la présente comme un eunuque, puis on parle d'elle comme d'un garçon, enfin, dans la dernière réplique du Duc, elle est un homme [a man], mais promise à un statut de reine [my fancy's queen] quand elle aura retrouvé ses habits de femme. À l'époque élisabéthaine, cela ne posait pas de problème particulier : Elisabeth elle-même en avait, paraît-il, donné l'exemple en haranguant ses troupes à Tilbury [1588], vêtue d'une cuirasse d'apparat: "J'ai le corps d'une faible femme mais le cœur d'un roi ». En 1962, rien de cela n'existait dans la conscience collective du public français, ni au reste la figure du " play boy", l'adolescent qui jouait les rôles de femme sur la scène élisabéthaine. Était-ce une gageure de jouer cette pièce en France? Non, il suffisait de confier le rôle de Viola à une comédienne très ravissante, qui restera femme tout au long de la pièce, malgré le discours des autres et le sien, une femme que la mise en scène présente d'emblée comme irrésistible: le Capitaine la couve d'un regard amoureux et la scène entre eux deux a une atmosphère très « flirt ». Et le tour est joué ! Encore fallait-il y penser.

11 Une grande économie de moyens mais, côté réflexion et travail scénique, un énorme investissement : le Troilus et Cressida présenté à la rue d'Ulm en 1974 me paraît la suite de tout cela. Certes, à ce point du raisonnement, quelqu'un pourrait protester: "Michèle, tu ne vas tout de même pas nous dire que c'est à l'École Normale qu'il faut aller chercher des sauvageons, des herbes folles, une esthétique en rupture d'avec les conventions et quoi encore ? Ni sur la scène ni - encore moins - dans la salle !»

12 Eh bien si ! À la fin des années 60, une réflexion s'était amorcée, du côté de la rue d'Ulm justement, sur le théatre universitaire : est-ce qu'on fait un peu la même chose que les professionnels, éventuellement en moins bien, ou est-ce qu'on choisit de faire ce que, pour des raisons diverses, eux ne peuvent pas faire? Quand on pose la question comme cela, la réponse coule de source. Pour l'Aquarium, à l'époque «troupe des Écoles Normales Supérieures ", explorer cette question a signifié se demander quelle pièce on allait écrire, et c'est ainsi que L'Héritier, d'après Bourdieu et Passeron, sera créée en 1968. Quelque chose qui ne fut pas explicité à l'époque m'apparaît plus nettement aujourd'hui : une troupe universitaire, qui ne verse pas de salaires, ne dépend pas de la presse, donc d'une critique favorable. On est dans l'artisanat, on bricole mais en travaillant dur; on n'a pas beaucoup de sous, mais côté fougue, on ne craint personne ; car on a à dire ce que personne ne dit. 
13 Dans ma mémoire, Troilus et Cressida de Stuart Seide, en 1974, correspond à cette formidable émergence. À lui de dire si mes souvenirs sont justes ou faux. Point important : on découvrait l'existence du spectacle en voyant une affiche. Car le théâtre universitaire supposait qu'on diffuse des affiches dans des lieux " amis », cela prenait des journées mais on le faisait de bon cœur. Ladite affiche annonçait que « La guerre et la luxure, il n'y a que cela qui soit toujours à la mode ", donc une mise en scène sous l'égide philosophique de Thersite. Pas de décor, peut-être même pas d'usage de la scène, seulement un proscenium qui s'avançait jusqu'au milieu du public comme une péninsule : comme indication du fait qu'on va à la rencontre du public, ce n'était pas mal. Des costumes simplifiés à l'extrême mais une présence physique forte des acteurs, lesquels s'amusaient comme des fous et vous faisaient entendre un texte, peut-être élagué mais d'une puissance cathartique rare. Ils étaient très bons ; la terreur et la pitié étaient au rendez-vous. Je ne sais plus si l'entrée était libre ou non mais j'ai la certitude qu'à la sortie, un jeune homme tenait un chapeau ; on vidait son porte-monnaie, en tout cas moi, et avec joie. Pourtant la pièce est dure, puisqu'il s'agit de l'exposition du «mensonge des idéaux» et de leur mise en déroute. Une fougue iconoclaste s'était montrée et avait su convaincre. Parfois je rêve d'un théâtre dont l'entrée serait libre, toujours, mais qui miserait sur l'honnêteté des spectateurs vis-à-vis du plaisir qu'ils viennent d'éprouver.

À Oxford en 1997, on ne passait pas le chapeau à la sortie du Songe d'une nuit d'été par The Northern Broadsides, à l'époque jeune compagnie fondée autour d'une révolte linguistique insigne - et qui savait communiquer le plaisir que l'on tire d'une libération. Jetez le carcan et votre énergie pourra s'épanouir dans la jubilation ludique. Des comédiens originaires du Nord de l'Angleterre et d'Écosse s'étaient un jour rebellés contre les écoles de théâtre qu'ils avaient fréquentées et ce qu'on avait essayé de leur imposer, à savoir la perte de leur accent régional. Zut à l'anglais du Sud-Est, norme qui nous écrase. Ils s'étaient mis ensemble pour jouer les classiques en gardant chacun le timbre de la région où il avait grandi. Et ils avaient tous un plaisir fou à faire résonner cette langue retrouvée, à en faire entendre la mélodie et les harmoniques de façon si claire qu'on se disait que sûrement c'était cet anglais-là, si coloré, si expressif, que l'on parlait jadis du côté de Stratford et chez les artisans de Londres, fort bien représentés dans la pièce par ceux d'Athènes. Et on comprenait tout, l'action, le texte, sans qu'il y ait jamais un geste de trop - ou parce qu'il n'y avait jamais un mouvement en trop. La question du "play boy " était astucieusement reprise : les fées étaient jouées par des malabars au pied léger. Je souhaite à tous les écoliers, et nous sommes tous des écoliers, de s'approprier les classiques de cette façon-là. Car les classiques sont là précisément pour qu'on se les approprie, qui qu'on soit.

\section{NOTES}

1. Alexandre Dumas, cité par Dominique Goy-Blanquet, Lettres à Shakespeare, Paris : éditions Thierry Marchaisse, 2014, p. 9. 
2. Voir «Entretien avec Aimé Césaire, 1975 », in Jacqueline Leiner, Aimé Césaire: le terreau primordial, Tübingen : Gunter Narr, 1993, vol. I, p. 134.

3. Charles Dullin, Ce sont les Dieux qu'il nous faut, Paris : Gallimard, 1969, p. 205, cité par Monique Surel-Tupin dans sa thèse (1979) sur Dullin, p. 112 (Charles Dullin, Bordeaux: Presses Universitaires de Bordeaux, 1984).

4. L'article est réédité dans Claude Francis et Fernande Gontier, Les Écrits de Simone de Beauvoir, Paris : Gallimard, 1979, p. 324.

5. Le journal s'intitule Action: organe social de la France combattante. Le numéro 1 est paru en octobre 1943. Le journal sort de la clandestinité avec son numéro 7 en juillet 1944. Simone de Beauvoir le décrit comme animé par un groupe de jeunes, mais c'est Francis Ponge qui se chargera de publier l'article.

6. In Écrits, op. cit., p. 326.

7. Augustin d'Humières, Homère et Shakespeare en banlieue, Paris : éditions Grasset, 2009. Pour les enfants d'un camp de réfugiés, voir l'article publié dans Le Parisien le 25 mars 2014. L'acteur syrien Nawwar Bulbul, voyant que ces enfants, privés de scolarisation, avaient même perdu le goût du jeu, eut l'idée de mettre en scène avec eux Le Roi Lear, en adaptant la pièce en arabe littéraire, une langue qui n'était pas vraiment la leur, ce qui imposait un effort d'apprentissage.

8. Simone de Beauvoir, La Force des choses, Paris : Gallimard 1963 ; Folio, 1972, t. I, p. 48-49.

9. In Écrits, op. cit., p. 291.

\section{RÉSUMÉS}

Cet article constitue le témoignage d'une spectatrice esthétiquement (et affectivement) engagée. Il cherche à montrer comment et pourquoi nous n'avons plus besoin de dire, comme Simone de Beauvoir en 1945, «C'est Shakespeare qu'ils n'aiment pas. »

This article recalls memories from an aesthetically and affectively involved female audience member. It aims at showing how and why we no longer need to say with Simone de Beauvoir, "It is Shakespeare they dislike".

\section{INDEX}

Keywords : Beauvoir Simone de, Dullin Charles, Macbeth, Midsummer Night's Dream (A), Shakespeare in France, Troilus and Cressida, Twelfth Night

Mots-clés : Beauvoir Simone de, Dullin Charles, Macbeth, Nuit des rois (La), Shakespeare en France, Songe d'une nuit d'été (Le), Troilus et Cressida

\section{AUTEUR}

MICHÈLE LE DOEUFF

CNRS 